\title{
Multi-Messenger analyses with the ANTARES High Energy Neutrino Telescope
}

\author{
Aurore Mathieu*, on behalf of the ANTARES Collaboration. \\ Centre de Physique des Particules de Marseille, Marseille, France \\ E-mail: amathieuecppm.in2p3.fr
}

\begin{abstract}
ANTARES is currently the largest neutrino telescope operating in the Northern Hemisphere, aiming at the detection of high energy neutrinos from astrophysical sources. Such observations would provide important clues about the processes at work in those sources, and possibly help solve the mystery of high energy cosmic rays. In this context, ANTARES has developed several multimessenger programs based on time and/or space coincidences with other cosmic probes such as photons, cosmic rays and gravitational waves. In this contribution, searches for neutrinos in correlation with blazars and microquasars, and TAToO, the follow-up program of ANTARES, are described.
\end{abstract}

Frontiers of Fundamental Physics 14

15-18 July 2014

Aix Marseille University (AMU) Saint-Charles Campus, Marseille, France

\footnotetext{
* Speaker.
} 


\section{Introduction}

The detection of astrophysical neutrinos and the identification of their sources is one of the main aims of large neutrino telescopes, like ANTARES in the Mediterranean Sea. Such observations would provide evidences for hadronic acceleration sites and so, for high energy cosmic rays sources.

As the atmospheric neutrino flux detected by ANTARES represents an irreducible background, several multi-messenger approaches have been developed. These methods allow to strongly reduce the background by searching for coincidences between different messengers in limited time/space windows. Different variable and transient sources can be observed in this way, such as gamma-ray bursts (GRBs) [1], core collapse supernovae (ccSNe) [2] or active galactic nuclei (AGNs).

In this contribution, searches for neutrino emission from microquasars and gamma-ray flaring blazars are presented, as well as a multi-messenger approach based on the follow-up of high energy neutrinos by a network of telescopes.

\section{The ANTARES neutrino telescope}

The ANTARES (Astronomy with a Neutrino Telescope and Abyss environmental RESearch) detector, completed in 2008, is located in the Mediterranean sea, $40 \mathrm{~km}$ off Toulon, France [3]. It consists of a tridimensional array of 885 photomultiplier tubes (PMTs) distributed on twelve $450 \mathrm{~m}$ high vertical detection lines. Each lines comprises up to 25 storeys hosting triplets of optical modules (OMs) oriented at 45 degrees with respect to the vertical, in order to optimize the detection of upgoing neutrino induced muon tracks. The geometry and size of the detector make it sensitive to neutrinos in the TeV-PeV range.

The neutrino detection relies on the emission of Cherenkov light by high energy muons originating from charged current neutrino interactions inside or near the instrumented volume. All detected signals are transmitted to a shore station via an optical cable, where data are filtered to search for coincident signals or hits in several adjacent OMs. The muon direction is then determined by maximising a likelihood function which compares the times of the hits with the expectation from the Cherenkov signal of a muon track.

\section{Time dependent search of neutrino emission from microquasars}

Microquasars are galactic X-ray binary systems exhibiting relativistic jets and are considered in some models to be possible sources of high energy (>100 GeV) neutrinos.

In this analysis, a search for neutrinos in coincidence with X-ray or $\gamma$-ray outbursts with 6 microquasars (Circinus X-1, GX 339-4, H 1743-322, IGRJ17091-3624, Cygnus X-1 and Cygnus X-3) was performed on 2007-2010 data [4].

The neutrino search for the four black hole binaries has been split in two kinds: observing the source during the hard X-ray states, and during the transition from hard to soft emission states, both scenarios when the acceleration could be dominated by hadronic acceleration. Samples of those period identifications are shown in fig. 1.

This X-ray emission information has been obtained from the Swift and ROSSI satellites. For the $\gamma$-ray bursts of Cygnus X-3, data from the Fermi LAT satellite has been used. The analyzed 


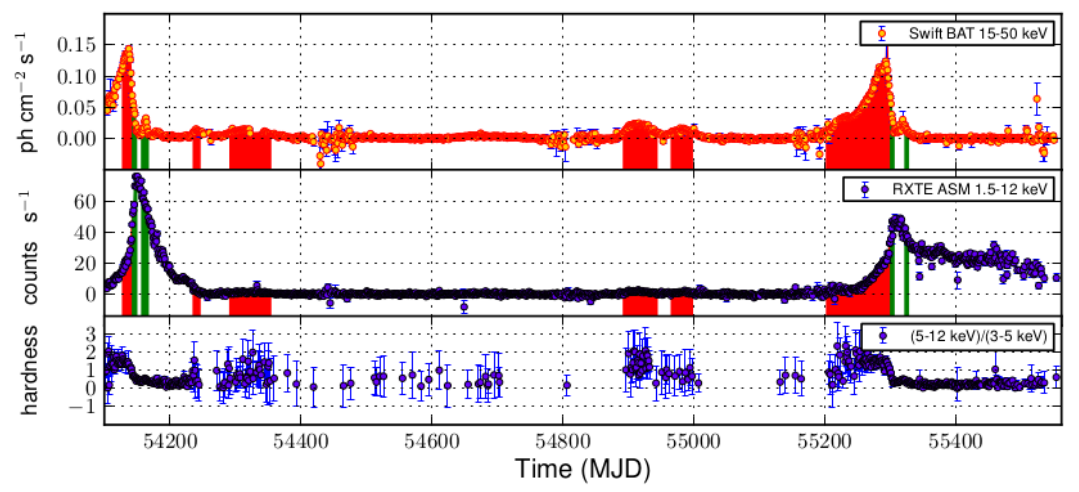

Figure 1: X-ray lightcurves of GX 339-4 between 2007 and 2010, where the identifications of the hard states are shown in the red filled areas and the hard to soft transition in the green filled areas.

period, by an unbinned method based on a likelihood ratio maximization, comprises the ANTARES data from 2007 up to 2010, a total of 813 days of live time. In this case, no event has been found in time coincidence, but upper limits have been computed for the given sources, as it is shown in table 1.

\begin{tabular}{|c|cc|}
\hline Source & Livetime (days) & Fluence U.L. \\
\hline Cir X-1 & 100.5 & 16.9 \\
GX 339-4 (HS) & 147.0 & 10.9 \\
GX 339-4 (TS) & 4.9 & 19.7 \\
H1743-322 (HS) & 84.6 & 9.1 \\
H1743-322 (TS) & 3.3 & 30.3 \\
IGRJ17091-3624 & 8.5 & 21.3 \\
Cyg X-1 (HS) & 182.8 & 14.1 \\
Cyg X-1 (TS) & 18.5 & 6.0 \\
Cyg X-3 & 16.6 & 5.7 \\
\hline
\end{tabular}

Table 1: List of the microquasars analyzed by ANTARES. The livetime is the effective time of observation of the source in days. Fluence is the Feldman-Cousins (90\% C.L.) upper limits on the neutrino fluence for a flux spectrum of $E_{v}^{2}$ in $\mathrm{GeV} \mathrm{cm}^{-2}$.

\section{Search for neutrino emission from gamma-ray flaring blazars}

Other transient sources of interest analyzed by ANTARES are active galactic nuclei. Of particular interest are the ones that show the highest brightness and variability, conformed by the so-called blazars subtypes BL Lac and FSRQ (Flat Spectrum Radio Quasar).

For this analysis [5], a search for neutrinos in coincidence with 41 bright and variable blazars chosen on the basis of their Fermi/LAT light curve profiles was performed using data from September 6th 2008 up to December 31st 2012 (total of 1044 days of live time).

For only three sources, 3C 279, PKS 1124-186 and PKS 0235-618, a neutrino signal has been 
found in coincidence with a gamma-ray flare. The most significant source is $3 \mathrm{C} 279$ with a pretrial p-value of $1.9 \%$, it has been found two high energy neutrino events. Figure 2 shows the time distribution of the Fermi gamma-ray light curve of 3C 279 with the time of the coincident neutrino events and the angular distribution around the position of this source. The post-trial probability computed taking into account the forty searches is $54 \%$ and is, thus, compatible with background fluctuations.
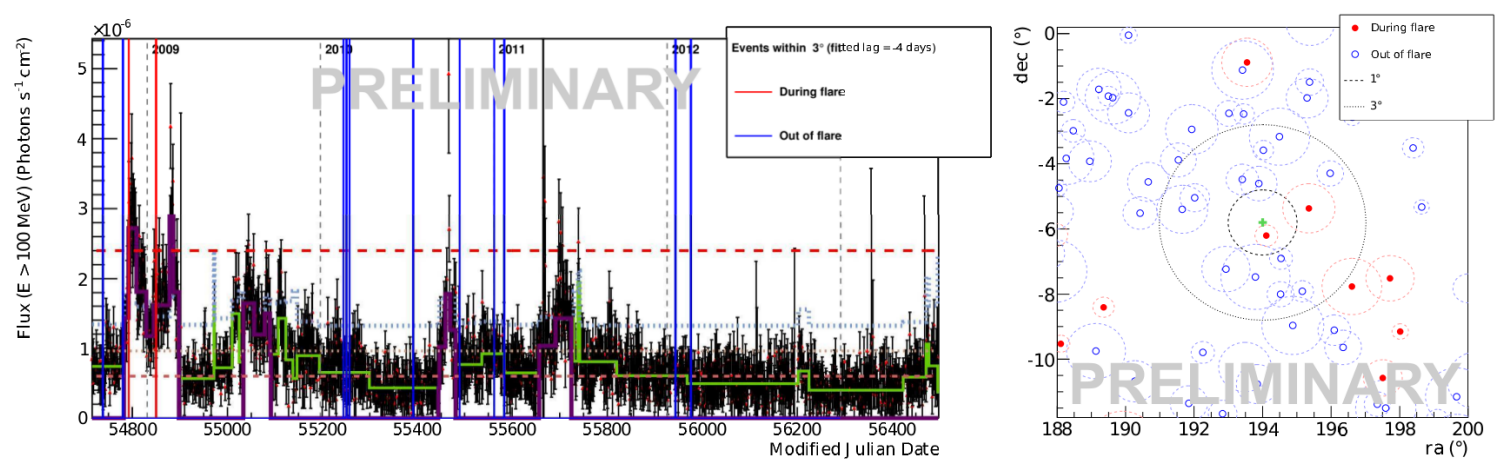

Figure 2: Left: Gamma-ray light curve (black dots) of the blazar 3C 279 measured by the LAT instrument above $100 \mathrm{MeV}$, with the selected neutrino events within $3^{\circ}$ around the source (red: events during the flare, blue: events out of the flare). The green histogram shows the denoised light curve. Right: Event map around the direction of 3C 279 (green cross) with two events compatible in time and direction (red dots). The blue empty dots represent the direction of all the selected events outside the flaring periods.

\section{TAToO: follow-up of high energy neutrinos}

In order to improve the sensitivity of ANTARES to transient sources, a detection method based on the follow-up of high energy neutrino alerts has been developed. A fast online muon track reconstruction is used to trigger a network of telescopes in case of special neutrino events. These events can be neutrino doublets coincident in space (within $3^{\circ}$ ) and time (within 15 minutes), single high energy neutrinos (typically above $5 \mathrm{TeV}$ ) or neutrinos coincident in space with a local galaxy (within $0.5^{\circ}$ ).

This method, described in [6], is sensitive to all transient sources producing high energy neutrinos, and the $2 \pi$ sr instantaneous sky coverage and the high duty cycle of ANTARES are important assets. The follow-up of interesting neutrino events possibly correlated to astrophysical transients can be done with fast-repositioning telescopes operating in different wavelenght domains. The network is composed of the small robotic optical telescopes TAROT, ROTSE and ZADKO and has been recently extended to the Swift/XRT telescope for X-ray follow-up to further improve the sensitivity to fast transient sources.

Alerts are sent on a regular basis of about 2 per month and images can be taken with a delay of $\sim$ $20 \mathrm{~s}$ after the neutrino alert, including the telescope slewing.

The observational strategy is composed of a real time observation followed by few observations up to two months after the neutrino alert. For example, a GRB afterglow requires a prompt observation as it decreases very quickly, contrary to ccSNe for which the optical signal will appear several 
days after the neutrino emission and can last several weeks.

Once the images are taken, an offline analysis can be performed with a dedicated pipeline composed of three main steps:

- Astrometric and photometric calibration

- Subtraction between an image and a reference which is a good quality image with no signal

- Candidates and lightcurves inspection

Since 2009, 136 alerts have been sent to our network with 5 alerts sent to the XRT telescope. Among these 136 alerts, 41 have prompt images (delay < 24h) which were analyzed. No transient optical counterpart associated to a neutrino alert was found in these images and upper limits on transient sources magnitude were derived. These upper limits can be applied to GRBs and a comparison with 158 optical afterglow lightcurves detected from 1997 to 2014 can be done (fig. 3). For images taken around 20 seconds after the neutrino alert, the presence of a GRB associated to a neutrino detection can be excluded with a probability higher than $95 \%$.

For the 5 alerts sent to Swift/XRT, no X-ray counterpart was found in correlation with a neutrino alert. We thus can compute upper limits on GRBs magnitude and compare with X-ray afterglow lightcurves detected by Swift/XRT (fig. 4).

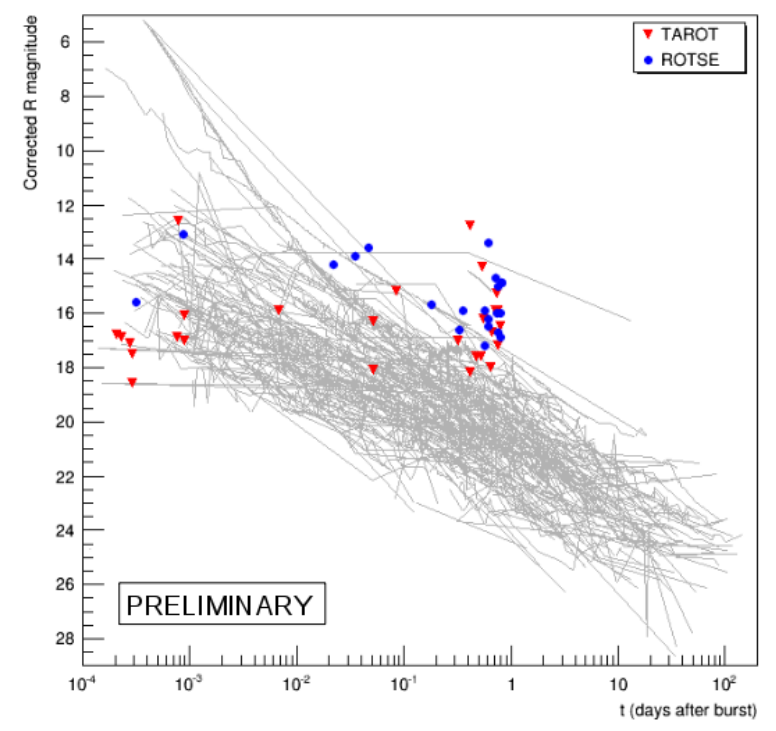

Figure 3: Grey: 158 optical afterglow lightcurves detected from 1997 to 2014. Red \& blue: upper limits on GRBs magnitude for TAROT and ROTSE alerts respectively.

\section{Conclusion}

Different multi-messenger analyses performed within the ANTARES Collaboration have been presented. The multi-messenger approach, based on space and/or time coincidences between different messengers, allows to significantly reduce the background for the cosmic neutrino search. The time information provided by different gamma-ray telescopes has been used to search for neutrino emission from microquasars and blazars. No event was found in time coincidence, but upper limits have been computed. 


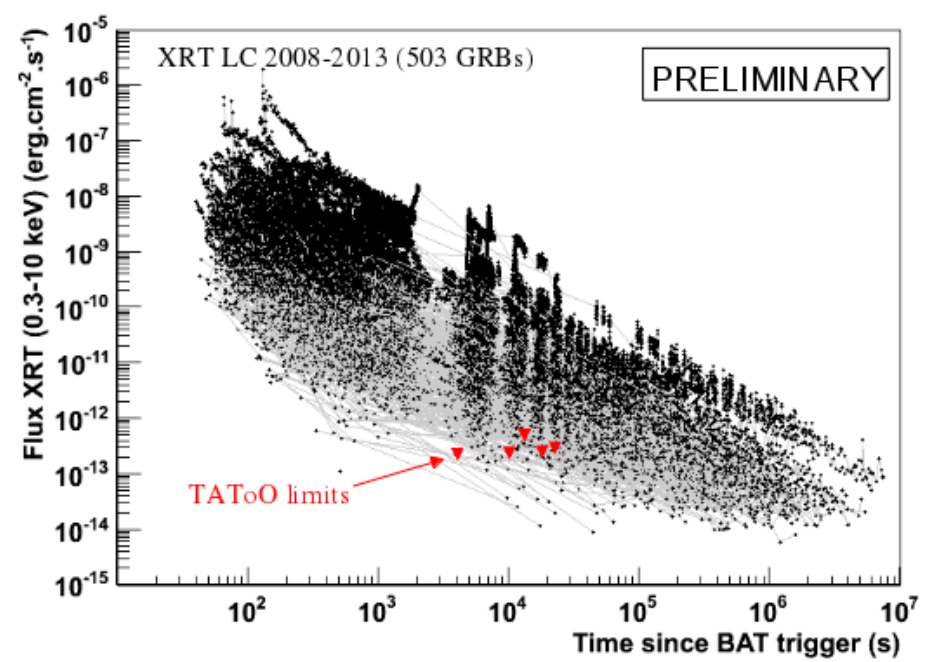

Figure 4: Grey: 503 X-ray afterglow lightcurves detected by Swift/XRT from 2008 to 2013. Red: upper limits on GRBs fluxes for 5 TAToO alerts.

And finally, TAToO, the follow-up program of high energy neutrinos has been described. The analysis of prompt images has not yet allowed to discover a transient source, in particular a GRB afterglow, associated to a neutrino alert. But, upper limits on the magnitude of a possible GRB afterglow have been set.

The analysis of follow-up images (delay $>24 \mathrm{~h}$ ) to search for a slowly rising signal, such as ccSNe, is ongoing.

\section{References}

[1] S. Razzaque, P. Meszaros, E. Waxman, Phys. Rev. Lett. 90 (2003) 241103

[2] S. Ando and J. Beacom, Phys. Rev. Lett. 95 (2005) 061103

[3] M. Ageron et al., ANTARES Collaboration, Nucl. Instrum. Meth. A 656 (2011) 11

[4] S. Adrián-Martinez et al., ANTARES Collaboration, J. of High Energy Astrophysics, 3 (2014) 9-17

[5] S. Adrián-Martinez et al., ANTARES Collaboration, Astropart. Phys. 36 (2012) 204210

[6] M. Ageron et al., ANTARES Collaboration, Astropart. Phys. 35 (2012) 530-536 